\title{
Limitations of microscopy to differentiate Plasmodium species in a region co-endemic for Plasmodium falciparum, Plasmodium vivax and Plasmodium knowlesi
}

Bridget E Barber ${ }^{1,2^{*}}$, Timothy William ${ }^{2,3}$, Matthew J Grigg ${ }^{1,2}$, Tsin W Yeo ${ }^{1,4}$ and Nicholas M Anstey $y^{1,4}$

\begin{abstract}
Background: In areas co-endemic for multiple Plasmodium species, correct diagnosis is crucial for appropriate treatment and surveillance. Species misidentification by microscopy has been reported in areas co-endemic for vivax and falciparum malaria, and may be more frequent in regions where Plasmodium knowlesi also commonly occurs.
\end{abstract}

Methods: This prospective study in Sabah, Malaysia, evaluated the accuracy of routine district and referral hospital-based microscopy, and microscopy performed by an experienced research microscopist, for the diagnosis of PCR-confirmed Plasmodium falciparum, P. knowlesi, and Plasmodium vivax malaria.

Results: A total of 304 patients with PCR-confirmed Plasmodium infection were enrolled, including 130 with $P$. knowlesi, 122 with $P$. falciparum, 43 with $P$. vivax, one with Plasmodium malariae and eight with mixed species infections. Among patients with $P$. knowlesi mono-infection, routine and cross-check microscopy both identified 94 (72\%) patients as "P. malariae/P. knowlesi"; 17 (13\%) and 28 (22\%) respectively were identified as P. falciparum, and $13(10 \%)$ and two (1.5\%) as P. vivax. Among patients with PCR-confirmed P. falciparum, routine and cross-check microscopy identified 110/122 (90\%) and 112/118 (95\%) patients respectively as $P$. falciparum, and 8/122 (6.6\%) and 5/118 (4.2\%) as "P. malariae/P. knowlesi". Among those with P. vivax, 23/43 (53\%) and 34/40 (85\%) were correctly diagnosed by routine and cross-check microscopy respectively, while 13/43 (30\%) and 3/40 (7.5\%) patients were diagnosed as "P. malariae/P. knowlesi". Four of 13 patients with PCR-confirmed P. vivax and misdiagnosed by routine microscopy as "P. malariae/P. knowlesi" were subsequently re-admitted with P. vivax malaria.

Conclusions: Microscopy does not reliably distinguish between $P$. falciparum, $P$. vivax and $P$. knowlesi in a region where all three species frequently occur. Misdiagnosis of $P$. knowlesi as both $P$. vivax and $P$. falciparum, and vice versa, is common, potentially leading to inappropriate treatment, including chloroquine therapy for $P$. falciparum and a lack of anti-relapse therapy for $P$. vivax. The limitations of microscopy in $P$. knowlesi-endemic areas supports the use of unified blood-stage treatment strategies for all Plasmodium species, the development of accurate rapid diagnostic tests suitable for all species, and the use of PCR-confirmation for accurate surveillance.

Keywords: Plasmodium knowlesi, Malaria, Microscopy, Diagnosis

\footnotetext{
*Correspondence: bridget.barber@menzies.edu.au

'Menzies School of Health Research and Charles Darwin University, Darwin,

Northern Territory, Australia

${ }^{2}$ Infectious Diseases Unit, Queen Elizabeth Hospital, Kota Kinabalu, Sabah,

Malaysia

Full list of author information is available at the end of the article
} 


\section{Background}

Despite recent progress towards elimination, malaria continues to affect over 200 million people per year with an estimated 655,000 deaths [1]. Although most deaths are caused by Plasmodium falciparum, the relative contribution of the non-falciparum Plasmodium species to the global malaria burden is increasing as incidence of $P$. falciparum falls [2-4]. The most widely distributed of these species is Plasmodium vivax, which accounts for half of the world's malaria and is increasingly recognized as a cause of severe and potentially fatal disease [5-8]. Moreover, reducing transmission of $P$. vivax has proved more difficult than P. falciparum, with successful malaria control programmes in some countries leading to an increase in incidence of $P$. vivax as overall malaria rates drop $[2,3]$. More recently, the simian parasite Plasmodium knowlesi has been identified as the most common cause of human malaria in parts of Malaysia [9-14], with its emergence also associated with reduction in incidence of the human Plasmodium species [12]. Plasmodium knowlesi is capable of causing severe disease and death [9,13,15-18], and is increasingly reported in other Southeast Asian countries [19].

In areas co-endemic for $P$. falciparum, $P$. vivax and $P$. knowlesi, species-differentiation at the time of diagnosis is crucial for directing appropriate treatment, particularly in settings which have separate treatment policies for different species, most commonly artemisinincombination treatment (ACT) for P. falciparum and chloroquine for non-falciparum species. Even in regions such as Papua, Indonesia, which have adopted a unified treatment strategy of ACT for all malaria [1], diagnosis of $P$. vivax is still required to allow administration of anti-hypnozoite treatment to prevent relapses, with misdiagnosis of this species potentially leading to increased morbidity and transmission. In areas also endemic for $P$. knowlesi, accurate diagnosis is important for epidemiological surveillance of this potentially fatal emerging zoonotic infection.

Microscopy of stained blood smears remains the standard method of malaria diagnosis in most parts of the malaria-endemic world, and ideally allows differentiation of species. However, with the difficulty in distinguishing young ring-stage parasites, frequent misdiagnosis has been reported in areas co-endemic for $P$. falciparum and $P$. vivax $[20,21]$. It is well established that microscopy cannot reliably distinguish $P$. knowlesi from Plasmodium malariae [22,23], but misdiagnosis of $P$. knowlesi with other species may also be frequent [13]. This study therefore evaluated the accuracy of both routine hospital microscopy and microscopy performed by an experienced research microscopist, for the diagnosis of $P$. falciparum, $P$. knowlesi, and $P$. vivax, in an area where all three species commonly occur.

\section{Methods}

\section{Study site and referral system}

The study was conducted at Queen Elizabeth Hospital (QEH), an adult tertiary referral hospital in Kota Kinabalu, Sabah, Malaysia. The hospital services the West Coast and Kudat Divisions of Sabah, with six district hospitals and a population of 1.14 million. From September 2010, in response to ongoing malaria deaths in Sabah [16], new treatment and referral guidelines were instituted and included tertiary hospital referral for all malaria patients with a thick blood film reported as " $4+$ " (indicating $>10$ parasites/high-power microscopy field [24]) or who had any evidence of severe malaria. Treatment was commenced prior to transfer and a pre-treatment blood film was sent with the patient. Local health clinics within the Kota Kinabalu area were required to refer all malaria patients to QEH for admission, with treatment commenced on arrival.

\section{Subjects}

All patients referred to or admitted directly to QEH with a microscopic diagnosis of malaria were assessed for eligibility from September 2010 to October 2011 as part of a prospective study of the epidemiology, clinical spectrum and pathophysiology of knowlesi malaria, reported elsewhere [15]. Non-pregnant patients $\geq 12$ years old were enrolled if they were within 18 hours of commencing malaria treatment, had no major co-morbidities, and had not already been enrolled in the study. Patients who were PCR negative were retrospectively excluded. Patients were classified as having severe malaria using modified 2010 WHO Severe Malaria Criteria [15,25,26]. Written informed consent was provided by patients or their relatives. Ethics approval was obtained from the Medical Research SubCommittee of the Malaysian Ministry of Health and the Health Research Ethics Committee of the Menzies School of Health Research, Australia.

\section{Study procedures}

Standardized data forms were used to record demographic and clinical information. Venous blood was collected in a CTAD tube labelled with the patient's study number, and thick and thin blood smears prepared using Giemsa staining. Species identification using thick and thin blood films was performed initially by microscopists at referring district hospitals, or at QEH if presenting directly to this hospital (routine microscopy). Thick and thin films were later cross-checked by a research microscopist (cross-check microscopy) with more than 15 years' experience, who was blinded to the results of routine microscopy. Because reliable differentiation of P. knowlesi from P. malariae is not possible [27], slides reported as $P$. malariae, $P$. knowlesi, or $P$. malariae/ $P$. knowlesi were all considered a single group, further 
referred to as "P. malariae/P. knowlesi". Parasite density was quantified by the research microscopist using pretreatment slides, and reported as the number of parasites per 200 white blood cells or per 1,000 red blood cells and converted to parasites $/ \mu \mathrm{L}$ using the patient's white blood cell count or haematocrit, respectively. If a pre-treatment slide could not be reliably obtained ( $6 \%$ of total slides) the referring hospital's microscopy report was used and the " $1+-4+$ " grade converted into parasites $/ \mu \mathrm{L}$ using the relevant median parasite density. Parasite DNA was extracted and PCR performed using previously described methods for $P$. falciparum, $P$. vivax, Plasmodium ovale, and P. malariae [28] and P. knowlesi [29]. PCR diagnosis was used as the gold standard. Patients were followed-up on days 14 and 28 if possible, and/or if readmitted to QEH.

\section{Statistical analysis}

Data were analysed using STATA version 10.1 (StataCorp LP, College Station, TX, USA). For continuous variables intergroup differences were compared using the Kruskal-Wallis test, or the Mann-Whitney test for pairwise comparisons, while the $X^{2}$ test was used for intergroup differences between categorical variables. Logistic regression was used to assess the association between parasite count and correct identification of species.

\section{Results}

A total of 304 patients with PCR-confirmed Plasmodium infection were enrolled, including 130 with $P$. knowlesi, 122 with $P$. falciparum, 43 with $P$. vivax, one with $P$. malariae, and eight with mixed-species infection. Demographic and clinical features are reported separately [15]. Half (51\%) of all patients were referred from district hospitals, including 86 (66\%) with knowlesi malaria, 47 (39\%) with P. falciparum, and 21 (49\%) with P. vivax malaria. Severe malaria occurred in 38 (29\%) patients with P. knowlesi, 13 (11\%) with P. falciparum, and seven (16\%) with $P$. vivax [15]. Patients with non-severe $P$. knowlesi had lower parasite counts than those with nonsevere P. falciparum $(4,837$ [IQR 1576-14,641] vs 10,500 [IQR 4,014-32,267] parasites/ $\mu \mathrm{L}, \mathrm{p}<0.01$ ), but not $P$. vivax (median 4,753 [IQR 2369-10,316] parasites/ $\mu \mathrm{L}$, $\mathrm{p}=0.95$ ). Among patients with severe malaria, there was no significant difference in median parasitaemia of those with P. knowlesi (80,359 [IQR 25,857-168,279] parasites $/ \mu \mathrm{L}$ ) and P. falciparum (72,270 [IQR 27,905273,909] parasites/ $\mu \mathrm{L}, \mathrm{p}=0.78$ ).

Microscopy and PCR results are shown in Tables 1 and 2. Slides were unavailable for cross-check microscopy for eight (2.6\%) patients. Routine and cross-check microscopy correctly identified the species in $229 / 304$ (75\%) and $242 / 296(82 \%)$ patients respectively $(\mathrm{p}=0.055)$, with 188 /
Table 1 PCR results compared with routine microscopy

\begin{tabular}{|c|c|c|c|c|c|c|c|c|}
\hline \multirow[t]{2}{*}{ Microscopy } & \multicolumn{8}{|c|}{ PCR } \\
\hline & $\mathrm{Pf}$ & $\mathrm{Pv}$ & $\mathrm{Pk}$ & Pm & $\mathrm{Pf} / \mathrm{Pv}$ & $\mathrm{Pf} / \mathrm{Pk}$ & $\mathrm{Pf} / \mathrm{Pm}$ & Total \\
\hline Pf & 110 & 3 & 17 & 0 & 2 & 2 & 0 & 134 \\
\hline Pv & 1 & 23 & 13 & 0 & 1 & 0 & 0 & 38 \\
\hline "Pm/Pk" & 8 & 13 & 94 & 1 & 0 & 0 & 1 & 117 \\
\hline "Pm/Pk"/Pv & 0 & 3 & 2 & 0 & 0 & 1 & 0 & 6 \\
\hline "Pm/Pk"/Pf & 2 & 0 & 3 & 0 & 0 & 0 & 0 & 5 \\
\hline $\mathrm{Pf} / \mathrm{Pv}$ & 1 & 1 & 0 & 0 & 1 & 0 & 0 & 2 \\
\hline "P species" & 0 & 0 & 1 & 0 & 0 & 0 & 0 & 1 \\
\hline Total & 122 & 43 & 130 & 1 & 4 & 3 & 1 & 304 \\
\hline
\end{tabular}

Abbreviations: $\mathrm{Pf}=$ Plasmodium falciparum, $\mathrm{Pv}=$ Plasmodium vivax, $\mathrm{Pk}=$ Plasmodium knowlesi, $\mathrm{Pm}=$ Plasmodium malariae, $\mathrm{P}=$ Plasmodium.

296 (64\%) patients correctly diagnosed by both microscopic methods. Among patients with PCR-confirmed $P$. knowlesi mono-infection, routine and cross-check microscopy each correctly identified $94(72 \%)$ patients as " $P$. malariae/P. knowlesi", with 66 (51\%) patients correctly diagnosed by both microscopy readings. Routine microscopy diagnosed 17 (13\%) and $13(10 \%)$ patients with PCRconfirmed $P$. knowlesi mono-infection as $P$. falciparum and $P$. vivax respectively, while cross-check microscopy diagnosed $28(22 \%)$ and two (1.5\%) as P. falciparum and $P$. vivax respectively. Four (3\%) patients with PCRconfirmed $P$. knowlesi were diagnosed by both routine and cross-check microscopy as $P$. falciparum, and no patient was diagnosed by both readings as $P$. vivax. Among the 38 patients with severe knowlesi malaria, routine microscopy correctly diagnosed 33 (87\%) as "P. malariae/P. knowlesi". Three were diagnosed as $P$. falciparum, one as $P$. vivax and one as P. falciparum/"P. malariae/P. knowlesi". The median parasite count among the five patients with severe knowlesi malaria and misdiagnosed by routine microscopy

\begin{tabular}{|c|c|c|c|c|c|c|c|c|}
\hline \multirow[t]{2}{*}{ Microscopy } & \multicolumn{8}{|c|}{ PCR } \\
\hline & $\overline{\mathrm{Pf}}$ & $\mathrm{Pv}$ & Pk & $\mathrm{Pm}$ & $\mathrm{Pf} / \mathrm{Pv}$ & $\mathrm{Pf} / \mathrm{Pk}$ & $\mathrm{Pf} / \mathrm{Pm}$ & Total \\
\hline$\overline{P f}$ & 112 & 0 & 28 & 0 & 1 & 3 & 0 & 144 \\
\hline $\mathrm{Pv}$ & 1 & 34 & 2 & 0 & 1 & 0 & 0 & 38 \\
\hline "Pm/Pk" & 5 & 3 & 94 & 1 & 0 & 0 & 1 & 104 \\
\hline $\mathrm{Pf} / \mathrm{Pv}$ & 0 & 1 & 2 & 0 & 1 & 0 & 0 & 4 \\
\hline Po & 0 & 2 & 0 & 0 & 0 & 0 & 0 & 2 \\
\hline Negative & 0 & 0 & $4^{*}$ & 0 & 0 & 0 & 0 & 4 \\
\hline Total & 118 & 40 & 130 & 1 & 3 & 3 & 1 & 296 \\
\hline
\end{tabular}

Abbreviations: $\mathrm{Pf}=$ Plasmodium falciparum, $\mathrm{Pv}=$ Plasmodium vivax, $\mathrm{Pk}=$ Plasmodium knowlesi, $\mathrm{Pm}=$ Plasmodium malariae, $\mathrm{Po}=$ Plasmodium ovale . Note: Slides were unavailable for cross-check microscopy for eight patients. Cross-check microscopy was performed on pre-treatment slides for 280 (95\%) of 296 patients.

*Includes one post-treatment slide. Parasite counts (according to routine microscopy results) of remaining three patients were 32, 55 and 1574 parasites/ $\mu \mathrm{L}$. 
was 168,279 (range 26,368 - 506,218) parasites $/ \mu \mathrm{L}$, and was not significantly different to those correctly diagnosed.

Among patients with PCR-confirmed P. falciparum, routine and cross-check microscopy correctly identified $110 / 122$ (90\%) and 112/118 (95\%) as P. falciparum, while $8 / 122(6.6 \%)$ and $5 / 118(4.2 \%)$ patients respectively were diagnosed as "P. malariae/P. knowlesi". All 13 patients with severe falciparum malaria were correctly diagnosed by routine microscopy.

Among those with PCR-confirmed P. vivax, 23/43 (53\%) and 34/40 (85\%) were correctly diagnosed by routine and cross-check microscopy respectively, while $13 / 43(30 \%)$ and $3 / 40(7.5 \%)$ patients were diagnosed as "P. malariae/P. knowlesi". Among the 13 patients with PCR-confirmed $P$. vivax and misdiagnosed by routine microscopy as "P. malariae/P. knowlesi", four (31\%) were re-admitted to QEH within four months with PCRconfirmed $P$. vivax infection. Among the seven patients with severe vivax malaria, routine microscopy diagnosed three as $P$. vivax, two as "P. malariae/P. knowlesi", one as $P$. falciparum/P. vivax, and one as " $P$. malariae/ $P$. knowlesi"/P. vivax.

Among the eight patients with mixed-species infection by PCR, one patient with $P$. falciparum/P. vivax was correctly identified by routine microscopy, and another with $P$. falciparum/P. vivax was correctly identified by cross-check microscopy. All others were misdiagnosed by both microscopic readings (with one patient's slide unavailable for cross-check microscopy).

An association was found between parasite count and correct identification of $P$. vivax by routine microscopy (OR [log increase] 1.64 [95\% CI $1.01-2.68], \mathrm{p}=0.046$ ), with a similar trend also seen with identification of $P$. vivax by cross-check microscopy (OR [log increase] 1.73 [95\% CI $0.96-3.10], \mathrm{p}=0.067$ ). No association however occurred between parasite count and correct identification of $P$. knowlesi or $P$. falciparum, by either routine or cross-check microscopy.

\section{Discussion}

This study highlights the difficulties of microscopic diagnosis of Plasmodium species in an area where P. falciparum, $P$. vivax and $P$. knowlesi all commonly occur. Misdiagnosis of $P$. knowlesi as both $P$. vivax and $P$. falciparum, and vice versa, is common. Only $72 \%$ of patients with PCR-confirmed P. knowlesi received an accurate diagnosis by routine or by cross-check microscopy, and correlation between the microscopic methods was poor, with even fewer patients receiving an accurate diagnosis of $P$. knowlesi by both methods. These findings occurred despite considering $P$. knowlesi and $P$. malariae as a single group, and so were not a consequence of the well described near impossibility of distinguishing these two species. Rather, patients with PCR-confirmed $P$. knowlesi were commonly misdiagnosed as having either $P$. falciparum or P. vivax malaria, with misdiagnosis of $P$. vivax as "P. malariae/P. knowlesi" also common.

The difficulty with distinguishing $P$. knowlesi from $P$. falciparum by microscopy has been previously described, due to similarities between the young rings of $P$. knowlesi and ring forms of $P$. falciparum, including double chromatin dots, multiple-infected erythrocytes and applique forms [22,23]. In a previous study in Sarawak, 11/216 (5\%) patients diagnosed by microscopy as "P. malariae" were actually $P$. falciparum by PCR, and 33/312 (11\%) microscopy-diagnosed P. falciparum cases were P. knowlesi by PCR [13]. In this previous study and in the current study, however, the difficulty differentiating $P$. knowlesi and $P$. vivax was also notable. In the current study $30 \%$ of patients with PCR-confirmed $P$. vivax were misdiagnosed by routine microscopy as " $P$. malariae/P. knowlesi", with four patients subsequently re-admitted with presumed vivax relapses due to lack of primaquine treatment. In the Sarawak study, 43 of 440 (10\%) patients with PCRconfirmed $P$. vivax malaria were misdiagnosed as " $P$. malariae" [13]. In a series of malaria deaths in Sabah, one of six fatal cases of $P$. knowlesi was misdiagnosed as $P$. vivax by microscopy and a fatal case of $P$. vivax was misdiagnosed as "P. malariae" [18].

This frequent misdiagnosis of $P$. vivax as " $P$. malariae/ $P$. knowlesi" has significant implications for malaria control, as failure to administer anti-hypnozoite treatment may lead to increased transmission and may hamper efforts to eliminate vivax malaria in regions where $P$. knowlesi is common. These findings support the current Sabah Ministry of Health policy for the performance of reference centre PCR on all patients with a microscopic diagnosis of "P. malariae/P. knowlesi" to enable administration of primaquine to those found to have misdiagnosed $P$. vivax. In knowlesi-endemic areas, where logistically possible, PCR should also be performed on at least a proportion of slides diagnosed as $P$. falciparum or $P$. vivax, to allow monitoring of the accuracy of microscopic diagnoses at different clinical sites, and to identify areas where additional training of microscopists may be required. Given the inaccuracies of microscopic diagnosis, performance of PCR is also essential to maintain accurate surveillance, particularly monitoring the emergence of $P$. knowlesi.

The inaccuracy of microscopy for differentiating Plasmodium species creates difficulties with basing treatment decisions on microscopic results. The 2008 Malaysian Ministry of Health malaria treatment guidelines recommend chloroquine for uncomplicated $P$. malariae/P. knowlesi malaria; chloroquine plus primaquine for uncomplicated $P$. vivax malaria; artemisinin combination treatment (ACT; artesunate/mefloquine [Artequine ${ }^{\circledR}$ ] or artemether/lumefantrine [Riamet $\left.{ }^{\circledR}\right]$ ) for 
uncomplicated P. falciparum malaria; and intravenous artesunate, or intravenous quinine plus oral doxycycline, for severe $P$. falciparum or severe $P$. malariae/P. knowlesi malaria [30]. No recommendations are given for treatment of severe vivax malaria. At Queen Elizabeth Hospital and referral hospitals in its catchment area, updated treatment guidelines recommend ACT for uncomplicated P. falciparum or P. malariae/P. knowlesi malaria; chloroquine plus primaquine, or ACT, for uncomplicated $P$. vivax malaria; and intravenous artesunate (followed by oral therapy as above) for severe malaria from any species [15].

Given the different treatment recommendations for each species in both of these guidelines, inappropriate treatment decisions may be made as a result of incorrect microscopic diagnoses. Misdiagnosis of $P$. falciparum as $P$. knowlesi would, under current national guidelines, result in administration of chloroquine for P. falciparum. Given widespread resistance of $P$. falciparum to chloroquine, this would lead to increased risk of complications and/or fatal outcome, as well as increased transmission. Patients with severe P. knowlesi malaria misdiagnosed as $P$. vivax may fail to receive immediate parenteral treatment if national guidelines are followed, and this scenario has been previously associated with fatal outcomes [18]. Even if the updated hospital guidelines are followed, misdiagnosis of severe $P$. knowlesi as $P$. vivax may still lead to treatment with oral chloroquine if signs of severity are missed, potentially leading to adverse outcomes given the slower parasite clearance associated with chloroquine as compared to oral ACT [16].

These results therefore support the argument for a unified treatment strategy of ACT for uncomplicated malaria from all Plasmodium species in knowlesi-endemic areas, an approach increasingly recommended for regions coendemic for $P$. falciparum and $P$. vivax [31]. These data also support the 2012 WHO recommendation for intravenous artesunate to be given to any patient meeting severe malaria criteria [25]. Even if signs of severity are overlooked among patients with knowlesi malaria, treatment with oral ACT may ensure more rapid parasite clearance and may lead to improved outcomes compared to treatment with oral chloroquine [16], although the optimal oral agent for uncomplicated P.knowlesi remains undetermined.

An additional advantage of this unified treatment approach would be the avoidance of inadvertent use of chloroquine for $P$. falciparum misdiagnosed as another species. Furthermore, chloroquine-resistant $P$. vivax is an increasing problem throughout Southeast Asia [31], and has been previously documented in Sabah [32] and Peninsular Malaysia [33-35]. Use of chloroquine for $P$. vivax malaria may therefore be associated with treatment failures, and may potentiate spread of chloroquine resistance.
Finally, this study highlights the need to develop rapid diagnostic tests (RDTs) that have the ability to distinguish between Plasmodium species, in order that reliable results can be obtained more quickly and cheaply than is possible with PCR. Although histidine-rich protein 2 (HRP2)-based RDTs are able to diagnose P. falciparum, RDTs that distinguish $P$. knowlesi from $P$. vivax are not yet available. Limited data suggest that P. knowlesi crossreacts with both $P$. falciparum and $P$. vivax-specific pLDH [36-39], and RDTs that combine these antigens with HRP2 may therefore allow differentiation between P. vivax, P. falciparum and P. knowlesi mono-infections. However, while a pLDH RDT has shown high sensitivity for the diagnosis of severe malaria from all three of these species, neither pLDH- or aldolase-based RDTs have demonstrated sufficiently high sensitivity for uncomplicated P. knowlesi [40]. Prospective evaluation of more sensitive RDTs in knowlesi-endemic areas is needed.

This study has found that microscopy does not reliably distinguish between $P$. falciparum, $P$. vivax and $P$. knowlesi in areas co-endemic for all three species, with misdiagnosis of $P$. vivax and $P$. knowlesi particularly common. In $P$. knowlesi-endemic areas these limitations of microscopic diagnosis must be considered when developing strategies to monitor the prevalence of the different malaria species, and when developing treatment guidelines.

\section{Competing interests}

The authors declare that they have no competing interests.

\section{Authors' contributions}

NMA, TWY, TW and BEB conceived and designed the study; BEB collected and analysed the data. BEB and NMA wrote the manuscript. MJG assisted with data collection. All authors read and approved the final manuscript.

\section{Acknowledgements}

We thank the clinical staff involved in patient care and microscopy staff at the district hospitals and QEH; Rita Wong, Beatrice Wong and Ann Wee for their help with clinical and laboratory study procedures; Ferryanto Chalfein for performing cross-check microscopy; Jutta Marfurt, Sarah Auburn and Nadine Kurz for performing the PCR assays; and the Director General of Health, Malaysia, for permission to publish this paper.

\section{Author details}

${ }^{1}$ Menzies School of Health Research and Charles Darwin University, Darwin, Northern Territory, Australia. ${ }^{2}$ Infectious Diseases Unit, Queen Elizabeth Hospital, Kota Kinabalu, Sabah, Malaysia. ${ }^{3}$ Sabah Department of Health, Kota Kinabalu, Sabah, Malaysia. ${ }^{4}$ Division of Medicine, Royal Darwin Hospital, Darwin, Northern Territory, Australia.

Received: 17 October 2012 Accepted: 20 December 2012 Published: 8 January 2013

\section{References}

1. World Health Organization: World Malaria Report 2011. Available: http:// www.who.int/malaria/world_malaria_report_2011/9789241564403_eng.pdf. Accessed 3rd May.

2. Nosten F, Van Vugt M, Price R, Luxemburger C, Thway K, Brockman A, McGready R, Ter Kuile F: Effects of artesunate-mefloquine combination on incidence of Plasmodium falciparum malaria and mefloquine resistance in western Thailand: a prospective study. Lancet 2000, 356:297-302.

3. Oliveira-Ferreira J, Lacerda MVG, Brasil P, Ladislau JLB, Tauil PL, Daniel-Ribeiro CT: Malaria in Brazil: an overview. Malar J 2010, 9:115. 
4. Gething PW, Elyazar IRF, Moyes CL, Smith DL, Battle KE, Guerra CA, Patil AP, Tatem AJ, Howes RE, Myers MF: A long neglected world malaria map: Plasmodium vivax endemicity in 2010. PLoS Negl Trop Dis 2012, 6:e1814.

5. Tjitra E, Anstey NM, Sugiarto P, Warikar N, Kenangalem E, Karyana M, Lampah DA, Price RN: Multidrug-resistant Plasmodium vivax associated with severe and fatal malaria: a prospective study in Papua Indonesia. PLoS Med 2008, 5:e128.

6. Genton B, D'Acremont V, Rare L, Baea K, Reeder JC, Alpers MP, Müller Plasmodium vivax and mixed infections are associated with severe malaria in children: a prospective cohort study from Papua New Guinea. PLoS Med 2008, 5:e127.

7. Price RN, Douglas NM, Anstey NM: New developments in Plasmodium vivax malaria: severe disease and the rise of chloroquine resistance. Curr Opin Infect Dis 2009, 22:430.

8. Anstey NM, Douglas NM, Poespoprodjo JR, Price R: Plasmodium vivax: clinical spectrum, risk factors and pathogenesis. Adv Parasitol 2012, 80:151-201.

9. Daneshvar C, Davis TM, Cox-Singh J, Rafa'ee M, Zakaria S, Divis P, Singh B: Clinical and laboratory features of human Plasmodium knowlesi infection. Clin Infect Dis 2009, 49:852-860.

10. Barber BE, William T, Dhararaj P, Anderios F, Grigg MJ, Yeo TW, Anstey NM: Epidemiology of Plasmodium knowlesi malaria in northeast Sabah Malaysia: family clusters and wide age distribution. Malar J 2012, 11:401

11. Barber BE, William T, Jikal M, Jilip J, Dhararaj P, Menon J, Yeo TW, Anstey NM: Plasmodium knowlesi malaria in children. Emerg Infect Dis 2011, 17:814-820

12. William T, Rahman HA, Jelip J, Ibrahim MY, Menon J, Grigg MJ, Yeo TW, Anstey NM, Barber BE: Increasing incidence of Plasmodium knowlesi malaria following control of $P$. falciparum and $P$. vivax malaria in Sabah, Malaysia. PLoS Negl Trop Dis 2013. In press.

13. Cox-Singh J, Davis TM, Lee KS, Shamsul SS, Matusop A, Ratnam S, Rahman HA, Conway DJ, Singh B: Plasmodium knowlesi malaria in humans is widely distributed and potentially life threatening. Clin Infect Dis 2008, 46:165-171.

14. Joveen-Neoh WF, Chong KL, Wong CM, Lau TY: Incidence of malaria in the Interior Division of Sabah, Malaysian Borneo, based on nested PCR. J Parasitol Res 2011, doi:10.1155/2011/104284.

15. Barber BE, William T, Grigg MJ, Menon J, Auburn S, Marfurt J, Anstey NM, Yeo TW: A prospective comparative study of knowlesi, falciparum and vivax malaria in Sabah, Malaysia: high proportion with severe disease from Plasmodium knowlesi and $P$. vivax but no mortality with early referral and artesunate therapy. Clin Infect Dis Advanced Access 2012. doi:10.1093/cid/cis902.

16. William T, Menon J, Rajahram G, Chan L, Ma G, Donaldson S, Khoo S, Fredrick C, Jilip J, Anstey NM, Yeo TW: Severe Plasmodium knowlesi malaria in a tertiary hospital, Sabah Malaysia. Emerg Infect Dis 2011, 17:1248-1255.

17. Cox-Singh J, Hiu J, Lucas SB, Divis PC, Zulkarnaen M, Chandran P, Wong KT, Adem P, Zaki SR, Singh B: Severe malaria-a case of fatal Plasmodium knowlesi infection with post-mortem findings. Malar J 2010, 9:10.

18. Rajahram G, Barber BE, William T, Menon J, Anstey NM, Yeo TW: Deaths due to Plasmodium knowlesi malaria in Sabah. Malaysia: association with reporting as P. malariae and delayed parenteral artesunate. Malar J 2012, 11:284.

19. Cox-Singh J: Zoonotic malaria: Plasmodium knowlesi, an emerging pathogen. Curr Opin Infect Dis 2012, 25. doi:10.1097/ OCO.1090b1013e3283558780.

20. Coleman RE, Maneechai N, Rachaphaew N, Kumpitak C, Miller RS, Soyseng V, Thimasarn K, Sattabongkot J: Comparison of field and expert laboratory microscopy for active surveillance for asymptomatic Plasmodium falciparum and Plasmodium vivax in western Thailand. Am J Trop Med Hyg 2002, 67:141-144.

21. McKenzies FE, Sirichaisinthop J, Miller RS, Gasser RA Jr, Wongsrichanalai C: Dependence of malaria detection and species diagnosis by microscopy on parasite density. Am J Trop Med Hyg 2003, 69:372-376.

22. Coatney GR, Collins WE, Warren MW, Contacos PG: The Primate Malarias. Washington: US Government Printing Office; 1971.

23. Lee K-S, Cox-Singh J, Singh B: Morphological features and differential counts of Plasmodium knowlesi parasites in naturally acquired human infections. Malar J 2009, 8:73.

24. World Health Organization: Basic Malaria Microscopy. Geneva: World Health Organization; 1991
25. World Health Organization: Management of severe malaria: a practical handbook. 3rd edition. Geneva: World Health Organization; 2012.

26. World Health Organization: Guidelines for the Treatment of Malaria. 2nd edition. Geneva: World Health Organization; 2010.

27. World Health Organization: Meeting Report: Informal Consultation on the Public Health Importance of Plasmodium knowlesi. Kuching, Sarawak, Malaysia: 2011

28. Padley D, Moody A, Chiodini P, Saldanha J: Use of a rapid, single-round, multiplex PCR to detect malarial parasites and identify the species present. Ann Trop Med Parasitol 2003, 97:131-137.

29. Imwong M, Tanomsing N, Pukrittayakamee S, Day NPJ, White NJ, Snounou G: Spurious amplification of a Plasmodium vivax small-subunit RNA gene by use of primers currently used to detect $P$. knowlesi. J Clin Microbiol 2009, 47:4173

30. Ministry of Health Malaysia: National antibiotic guideline 2008. Available: http://www.pharmacy.gov.my/aeimages//File/ National_Antibiotic Guideline_2008_edit\%282\%29.pdf. Accessed 3rd August 2012

31. Douglas NM, Anstey NM, Angus BJ, Nosten F, Price RN: Artemisinin combination therapy for vivax malaria. Lancet Infect Dis 2010, 10:405-416

32. Ahlm C, Wiström J, Carlsson H: Chloroquine resistant Plasmodium vivax malaria in Borneo. J Travel Med 1996, 3:124-124.

33. Baird JK: Chloroquine resistance in Plasmodium vivax. Antimicrob Agents Chemother 2004, 48:4075-4083.

34. Jamaiah I, Rohela M, Nissapatorn V, Amriana A, Sumaiyah M, Lila M, Norzawati A: Malaria: A retrospective study in Hospital Tengku Ampuan Rahimah (HTAR), Klang, Selangor, Malaysia (2004-2006). Southeast Asian J Trop Med Public Health 2007, 38:1.

35. Jamaiah I, Rohela M, Nissapatorn V, Khoo B, Khoo P, Radhiyah M, Aisyah A: Malaria: a 10-year (1994-2003) retrospective study at University Malaya Medical Center (UMMC), Kuala Lumpur, Malaysia. Southeast Asian J Trop Med Public Health 2005, 36:60-63.

36. Tang T, Salas A, Ali-Tammam M, Martinez M, Lanza M, Arroyo A, Rubio J: First case of detection of Plasmodium knowlesi in Spain by Real Time PCR in a traveller from Southeast Asia. Malar J 2010, 9:219.

37. van Hellemond J, Rutten M, Koelewijn R, Zeeman A, Verweij J, Wismans P, Kocken C, van Genderen P: Human Plasmodium knowlesi infection detected by rapid diagnostic tests for malaria. Emerg Infect Dis 2009, 15:1478.

38. Kawai S, Hirai M, Haruki K, Tanabe K, Chigusa Y: Cross-reactivity in rapid diagnostic tests between human malaria and zoonotic simian malaria parasite Plasmodium knowlesi infections. Parasitol Int 2009, 58:300-302.

39. McCutchan T, Piper R, Makler M: Use of malaria rapid diagnostic test to identify Plasmodium knowlesi infection. Emerg Infect Dis 2008, 14:1750.

40. Barber BE, William T, Grigg M, Piera KA, Yeo TW, Anstey NM: Evaluation of the sensitivity of a pLDH-based and an aldolase-based Rapid Diagnostic Test for the diagnosis of uncomplicated and severe malaria caused by PCR-confirmed Plasmodium knowlesi, P. falciparum and P. vivax. under review.

\section{doi:10.1186/1475-2875-12-8}

Cite this article as: Barber et al:: Limitations of microscopy to differentiate Plasmodium species in a region co-endemic for Plasmodium falciparum, Plasmodium vivax and Plasmodium knowlesi. Malaria Journal 2013 12:8.

\section{Submit your next manuscript to BioMed Central and take full advantage of:}

- Convenient online submission

- Thorough peer review

- No space constraints or color figure charges

- Immediate publication on acceptance

- Inclusion in PubMed, CAS, Scopus and Google Scholar

- Research which is freely available for redistribution 